\title{
Análisis del impacto de la mortalidad por homicidios de acuerdo al Índice de Necesidades Básicas Insatisfechas en Colombia, 2000-2011*
}

\section{Analysis of the Impact of the Homicide Mortality Rate According to the Unmet Basic Needs Index in Colombia 2000-20II}

\section{Análise do impacto da mortalidade por homicídio segundo o Indicador de Necessidades Básicas Não Satisfeitas na Colômbia, 2000-20II}

Fecha de recepción: 28-01-15 Fecha de aceptación: 16-03-15 Disponible en línea: 30-06-15 doi: 10.11144/Javeriana.rgyps18-28.aimh

Como citar este artículo:

Dávila-Cervantes CA, Pardo-Montaño, AM. Análisis del impacto de la mortalidad por homicidios de acuerdo al Índice de Necesidades Básicas Insatisfechas en Colombia, 2000-2011. Rev. Gerenc. Polít. Salud. 2015; 14(28): 63-77. http://dx.doi.org/10.11144/Javeriana.rgyps18-28.aimh

*** Autora de correspondencia. Doctora en Geografía, investigadora asociada del Instituto de Geografía de la Universidad Nacional Autónoma de México. Universidad Nacional Autónoma de México, Circuito de la Investigación Científica, Ciudad Universitaria, 04510, Coyoacán, México. Correo electrónico: apardo@igg.unam.mx. 


\section{Resumen}

El objetivo fue analizar la mortalidad por homicidios en Colombia entre 2000 y 2011, estratificando cada departamento por el Índice de Necesidades Básicas Insatisfechas (INB). Se utilizaron estadísticas de mortalidad, calculando tasas estandarizadas y años de vida perdidos (AVP) entre 15 y 49 años, bajo el supuesto de mortalidad nula. Existió un incremento de la mortalidad en el periodo 2000-2002; del 2003 al 2011 hubo una reducción importante. La tasa de mortalidad disminuyó 48\% (50\% hombres, 39\% mujeres); los AVP se redujeron $16 \%$ (16\% hombres y $14 \%$ mujeres). Respecto al INB, el estrato medio presentó la mayor mortalidad; el bajo la disminución más importante. En mujeres el estrato medio tuvo los mayores AVP; en hombres, el alto presentó los menores. Se corroboró el descenso de la mortalidad por homicidios en Colombia. Es vital proveer insumos para elaborar políticas de prevención de la mortalidad por homicidios a partir de investigaciones que permitan tener una visión integral del fenómeno.

Palabras clave: mortalidad; violencia; homicidio; esperanza de vida; tasa de mortalidad; Colombia

\section{Abstract}

The objective was to analyze the mortality due to homicides in Colombia, during the years 2000 to 2011, stratifying each department according to the Unmet Basic Needs Index (INB). We used mortality statistics by calculating standard rates and years of life expectancy lost (AVP) between ages 15 and 49 , under the assumption of null mortality. There was an increase in mortality during the period 2000-2002; from 2003 to 2011 there was an important decrease. The mortality rate dropped $48 \%$ ( $50 \%$ for men, $39 \%$ for women); the AVP dropped $16 \%$ ( $16 \%$ for men, $14 \%$ for women). Regarding the INB, the middle socio-economic status showed the highest mortality; the low socio-economic status showed the highest drop. Women in the middle socio-economic status had the highest AVP score; men from the high socio-economic status had the lowest AVP scores. We confirmed a reduction in the mortality rate due to homicides in Colombia. It is vital to provide supplies for the creation of policies aimed at the prevention of mortality due to homicides, by means of research that allow an integral vision of this phenomenon.

Keywords: mortality; violence; life expectancy; mortality rate; Colombia

\section{Resumo}

O objetivo foi analisar a mortalidade por homicídios na Colômbia entre 2000 e 2011, estratificando cada departamento através do Indicador de Necessidades Básicas Não Satisfeitas (INB). Estatísticas de mortalidade foram utilizadas, calculando taxas padronizadas e anos de vida perdidos (AVP) entre 15 e 49 anos, sob o suposto de mortalidade nula. Houve acréscimo da mortalidade no período 2000-2002; do 2003 a 2011 houve redução importante. A taxa de mortalidade diminuiu $48 \%$ (50\% homens, $39 \%$ mulheres); os AVP reduziram-se $16 \%$ (16\% homens e $14 \%$ mulheres). No que diz respeito do INB, a classe média apresentou maior mortalidade; a baixa, a diminuição mais importante. Em mulheres, a classe média teve os maiores AVP; em homens, a alta apresentou os menores. Corroborou-se a descida da mortalidade por homicídios na Colômbia. Prover insumos é vital para elaborar políticas de prevenção da mortalidade por homicídio a partir de pesquisas que permitam ter uma visão integral do fenômeno.

Palavras-chave: mortalidade; violência; homicídio; expectativa de vida; taxa de mortalidade; Colômbia 


\section{Introducción}

La violencia es un tema complejo dadas sus características y consecuencias, y es actualmente uno de los principales problemas de salud pública a nivel mundial (1-3). La violencia se manifiesta de diferentes maneras, encontrando en el homicidio una forma extrema de expresión de graves problemas sociales, tanto porque se priva a la víctima de la totalidad de sus derechos, como por el profundo significado social que tiene (4-6).

A nivel mundial, en el año 2000 se registraron 520000 homicidios, aproximadamente, lo que representó una tasa de mortalidad de 8,8 homicidios por 100000 habitantes (2), cifra que disminuyó para 2012, con un número global estimado de homicidios de 437000 (con una tasa de 6,2 por 100000 habitantes) (7). Se calcula, además, que del total de homicidios, $36 \%$ ocurrieron en el continente americano (con una tasa superior a 16 por $100000)(7,8)$. Por edad, las tasas más altas correspondieron al grupo de 15 a 29 años, seguido por el de 30 a 49 años. Resalta que más de $90 \%$ de los homicidas y víctimas son hombres, y casi la mitad de todas las víctimas tienen entre 15 y 29 años de edad, y poco menos de un tercio se encuentran entre los 30 y los 44 años $(4,7,9)$. Otra característica de los homicidios en el mundo es que existe una brecha cada vez mayor en los niveles entre los países con tasas altas y aquellos con tasas bajas.

Entre los países de la región, Colombia es considerado uno de los más violentos. A principios de los años ochenta se presentó una escalada de la violencia, la cual se intensificó a partir de 1984, año en el cual la tasa de mortalidad se encontraba en un nivel de aproximadamente 40 homicidios por cada 100000 habitantes; este incremento continuó hasta 1991, cuando alcanzó su máximo nivel, de aproximadamente 85 homicidios por cada
100000 habitantes $(10,11)$. A partir de ese año se presenta una disminución paulatina de la tasa, regresando a niveles inferiores a 60 homicidios en 1997 (11). Sin embargo, en el siguiente lustro, la mortalidad por esta causa se incrementó nuevamente, alcanzando un nivel superior a 75 homicidios (11).

Por tanto, en las últimas tres décadas, los homicidios, junto con otras formas de violencia, han sido el principal problema social, económico, de salud pública y de seguridad en Colombia (12). Esto ha justificado la realización de diversos estudios que han intentado establecer las causas de la violencia homicida en el país. Se destacan aquellos que han investigado sobre la relación entre los factores económicos, como la pobreza, la desigualdad y el crecimiento económico, con la violencia (13-15). Por otro lado, otros estudios se han enfocado en cuestiones culturales, en un débil control estatal y en la presencia y actividad de grupos armados irregulares, como variables explicativas de la violencia homicida $(16,17)$. Considerando esto, cabe preguntarse, de manera general, ¿cuál es la relación entre la mortalidad por homicidios y la pobreza en el caso colombiano en los últimos años?, ¿cuál es su impacto en la esperanza de vida?, ¿qué diferencias se observan a nivel departamental?

Dentro de este marco, el objetivo principal de esta investigación es analizar la mortalidad por homicidios en Colombia entre el 2000 y el 2011, los niveles, su tendencia y el impacto en la esperanza de vida, realizando una estratificación a nivel departamental por medio del Índice de Necesidades Básicas Insatisfechas (INB), por sexo, en el grupo de edades de 15 a 49 años, y utilizando para ello las tasas estandarizadas de mortalidad y los años de vida perdidos (AVP).

Se realiza el estudio por sexo, debido a que en el análisis de la mortalidad por homici- 
dios esta diferenciación por género es muy importante, pues tanto las víctimas como los victimarios son mayoritariamente hombres $(8,18)$. Debido a que en algunos estudios se ha encontrado una relación entre pobreza y violencia homicida en Colombia $(4,15)$, y dado que desde finales de los años ochenta en el país la pobreza ha sido medida con la metodología de Necesidades Básicas Insatisfechas (19), se utilizó este indicador para analizar la posible relación entre la mortalidad por homicidios y la pobreza, y con base en ello aportar mayor evidencia en torno a este debate.

\section{Material y métodos}

Se realizó un estudio descriptivo, comparativo y transversal, con información proveniente de las estadísticas vitales del Departamento Administrativo Nacional de Estadística (DANE) en Colombia. Los datos de defunciones se obtuvieron para el periodo 2000-2011, por departamento, sexo y por grupos quinquenales de edad. La población a mitad de año proviene de las estimaciones de población del DANE. Se adoptó la definición de homicidio de la Clasificación Internacional de Enfermedades (CIE-10), la cual abarca los códigos X85-Y09 y Y87.1. Para la estratificación socioeconómica se utilizó el Índice de Necesidades Básicas Insatisfechas (INB), calculado a nivel departamental por el DANE (basado en el Censo de Población de 2005 y actualizado al 2011). Para el cálculo de los AVP se utilizaron las tablas de vida nacionales, por sexo, del periodo 20052010, provenientes de las proyecciones de población del DANE (2005-2020).

Para medir la violencia, en este estudio se trabaja con los homicidios, debido tanto a la gravedad del acto como a que es el indicador de violencia que se registra con mayor 66 precisión y periodicidad (20). Asimismo, se excluyeron del análisis las defunciones por homicidios en cuyo registro no constaban la edad ni el sexo, quedando descartados un $2,4 \%$ del total. Para esta investigación se consideraron los 15 a 49 años de edad como límites para el cálculo de los AVP, debido a que en Colombia los homicidios son una de las principales causas de muerte en este rango etario, lo cual se refleja en que desde el 2000 se ha concentrado más del $85,3 \%$ del total de homicidios en esas edades, con un máximo de $87,5 \%$ en el 2001. Al calcular los AVP no se contabilizaron las defunciones registradas en los menores de 15 y en los mayores de 49 años de edad (30909 casos), por lo que se descartaron $12,5 \%$ del total de casos.

Las tasas estandarizadas de mortalidad por homicidios, por grupo de INB, sexo y grupos quinquenales de edad para el periodo abarcado por el estudio, se calcularon utilizando la población nacional del año 2005 como estándar. Se calcularon los AVP propuestos por Arriaga $(21,22)$, para estimar cuántos años de vida en promedio se perdieron por homicidios entre los 15 y los 49 años de edad. Se utilizó el supuesto de mortalidad nula entre las dos edades elegidas $(21,22)$. Esta técnica es considerada además como una de las principales herramientas para medir los cambios en el nivel de la mortalidad y la mortalidad por causas; asimismo, es un indicador útil que permite relacionar la mortalidad por homicidios con el cambio de la esperanza de vida temporaria o entre edades seleccionadas (22), dando cuenta de los cambios y del impacto de la mortalidad por esta causa en la salud de la población (23). Este procedimiento tiene la ventaja de que al comparar la mortalidad observada con la mortalidad hipotética nula, las comparaciones quedan estandarizadas y por lo tanto se pueden realizar comparaciones históricas o entre poblaciones fácilmente (22).

El INB se utiliza para estimar la magnitud de la pobreza en relación con la población, 
se asume que las personas que habitan en viviendas con INB o en situación de miseria se encuentran en las mismas condiciones de su respectiva vivienda (24). El índice se calcula por medio de cinco indicadores simples seleccionados de las viviendas clasificadas como: inadecuadas, con hacinamiento crítico, con servicios inadecuados, con alta dependencia económica, con niños en edad escolar que no asisten a la escuela (24). El índice se mide en valores porcentuales, indicando con un $100 \%$ el número más alto de necesidades insatisfechas de la población; este indicador osciló entre 9,2\% (Bogotá) y 79,2\% (Chocó) en el 2011. Este método ha significado un importante aporte para la identificación de ciertas carencias críticas de la población y para la caracterización de la pobreza, ya que provee la capacidad para: i) incluir o excluir beneficiarios de las políticas contra la pobreza, ii) hacer comparaciones en el tiempo, iii) hacer comparaciones en el espacio, iv) hacer diagnósticos sociales, v) apoyar la puesta en práctica de programas sociales y vi) completar las mediciones de la pobreza por ingresos y ofrecer alternativas para subsanar las fallas de su aplicación en lo que respecta a disponibilidad de servicios básicos y condiciones de las viviendas (19). A partir de este indicador se hizo un análisis de distribución por quintiles, con el propósito de obtener cinco estratos: Muy Bajo, Bajo, Medio, Alto y Muy Alto (tabla 1).

\section{Resultados}

\section{Análisis a nivel nacional}

En el año 2000, los homicidios representaban alrededor de $15,4 \%$ del total de muertes registradas (con un máximo de 16,5\% en el 2002), porcentaje que disminuyó a $8,5 \%$ en el 2011. La tasa estandarizada de mortalidad por homicidios entre el 2000 y el 2002 presentó un incremento de 71,3 a 76,7 homicidios por cada 100000 habitantes (lo que equivale un aumento de 7,5\%); posteriormente, la mortalidad desciende de manera gradual, hasta alcanzar un valor de 36,1 por 100000 en el 2011 (lo que representó una disminución de 52,9\% con respecto al 2002). En el caso de hombres y mujeres se observa una tendencia análoga; esto es, entre el 2000 y el 2002 se presentó un incremento de las tasas (6,4\% para hombres y $17,7 \%$ para mujeres)

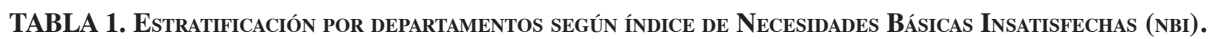
Colombia, 2011

\begin{tabular}{|c|c|l|}
\hline $\begin{array}{c}\text { NBI (número de departamentos } \\
\text { en el estrato) }\end{array}$ & $\begin{array}{c}\text { Rango de cada estrato } \\
(\mathbf{\%})\end{array}$ & \multicolumn{1}{|c|}{ Departamentos } \\
\hline Muy Alto (6) & $54,9-79,2$ & $\begin{array}{l}\text { Chocó, Córdoba, Guainía, La Guajira, } \\
\text { Sucre, Vichada }\end{array}$ \\
\hline Alto (7) & $42,6-54,8$ & $\begin{array}{l}\text { Amazonas, Bolívar, Cesar, Cauca, Magda- } \\
\text { lena, Nariño, Vaupés }\end{array}$ \\
\hline Medio (7) & $31,9-42,5$ & $\begin{array}{l}\text { Arauca, Caquetá, Casanare, Guaviare, } \\
\text { Huila, Putumayo, San Andrés, }\end{array}$ \\
\hline Bajo (7) & $21,8-31,8$ & $\begin{array}{l}\text { Antioquia, Atlántico, Boyacá, Meta, Norte } \\
\text { de Santander, Santander, Tolima }\end{array}$ \\
\hline Muy Bajo (6) & $9,2-21,7$ & $\begin{array}{l}\text { Bogotá*, Caldas, Cundinamarca, Quindío, } \\
\text { Risaralda, Valle del Cauca }\end{array}$ \\
\hline
\end{tabular}

* Se consideró como un conglomerado más por ser la capital del país.

Fuente: elaboración a partir del índice de Necesidades Básicas Insatisfechas a nivel departamental, Colombia, 2005, Departamento Administrativo Nacional de Estadística (DANE). 


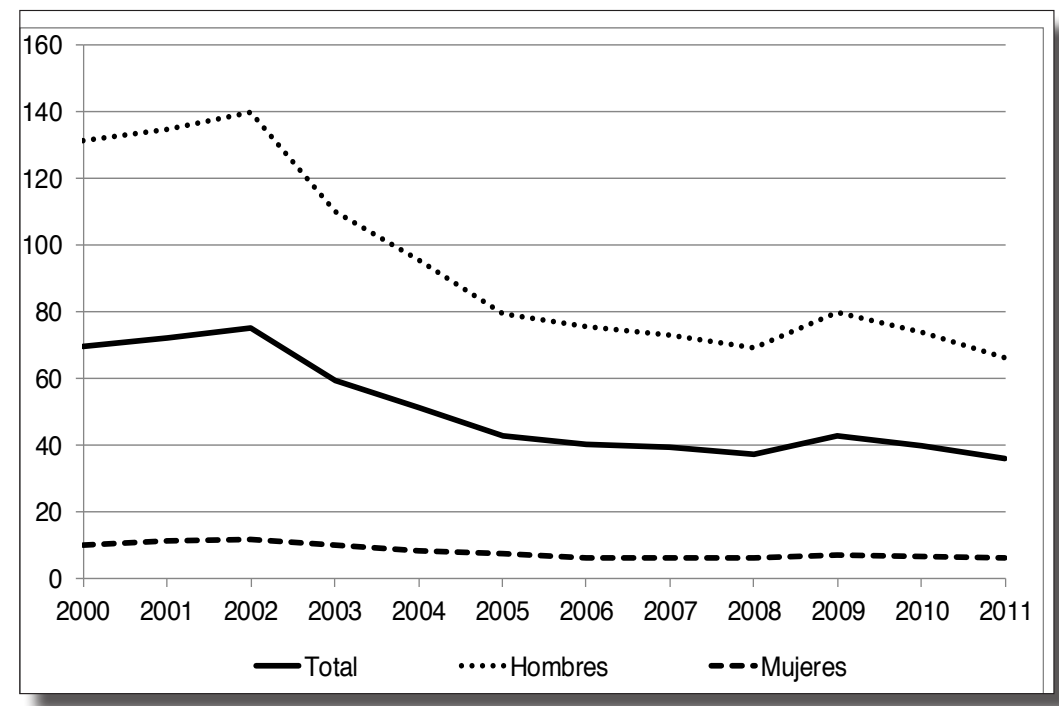

Figura 1. Tasas de mortalidad por homicidios, Colombia 2000-2011

Fuente: elaboración propia con base en datos del DANE, 2000-2011

(figura 1), mientras que en el periodo siguiente (2002-2011) se dio una disminución significativa de la tasa $(53,7 \%$ en hombres y $48,7 \%$ para mujeres), alcanzando en el 2011 un nivel de 66,2 y 6,1 homicidios para hombres y mujeres, respectivamente.

\section{Análisis por edad}

Los AVP en el grupo de edad considerado, entre el año 2000 y el 2002, aumentaron un 5,1\% (4,9\% para hombres y $20,3 \%$ para mujeres), alcanzando en el 2002 un valor máximo de 0,58 años para el total, 1,03 en hombres y de 0,1 años en mujeres, lo que implica que el impacto de la mortalidad por homicidios en la esperanza de vida temporaria masculina entre los 15 y 49 años de edad fue una reducción en poco más de un añoy en las mujeres de 0,1 años (tabla 2). A partir del 2002 los AVP disminuyeron gradualmente hasta el 2011, con un cambio relativo de $22,2 \%$ en ambos sexos $(19,5 \%$ en hombres y $28,8 \%$ en mujeres), el cual es menor al observado en las tasas debido a que esta caída de la mortalidad se produjo, en mayor medida, en los mayores de 35 años de edad, característica observada en otra investigación alrededor del tema (25). Cabe mencionar también que en el 2009 se presentó un repunte de la mortalidad por esta causa, aumentando un $10,7 \%$ con respecto al año anterior; el cambio para hombres fue de $9,3 \%$ y de $13,5 \%$ para mujeres. Es importante resaltar que cuando se compara el cambio relativo de las tasas de mortalidad y de los AVP, la situación es distinta; mientras que la tasa disminuyó en poco más de $48 \%$ en ambos sexos (casi $50 \%$ para hombres y poco más de 39\% en mujeres), entre el 2000 y el 2011 los AVP en el grupo de edad de 15 a 49 años se redujeron en un monto considerablemente menor en términos relativos (16,2\% en ambos sexos, $15,6 \%$ en hombres y $14,2 \%$ en mujeres). Esto se puede deber a que el cambio relativo de la mortalidad por homicidios fue mayor en edades de 35 años y más, y no tan significativo en las más jóvenes, por lo que no existió una disminución tan marcada de AVP (tabla 2).

\section{Análisis por estrato de INB}

La tasa de homicidios por estrato de INB, en general, presentó una tendencia a disminuir 
entre el 2000 y el 2011. En el 2000, el estrato con la mayor tasa de mortalidad por homicidios era el de Bajo INB, con un nivel de 82 homicidios, mientras que el que presentaba las menores tasas era el de Muy Alto INB, con una tasa de 38,8 homicidios. En el periodo 2000-2002 se observó también el aumento de las tasas, previamente mencionado, siendo el estrato de INB Medio el que presentó los mayores incrementos de la tasa, con un aumento de $50 \%$, superando una tasa de 114 homicidios para ambos sexos (y mayor a 200 en hombres y 24 para mujeres) en el 2002 (figuras 2 y 3). A partir de ese año se da un descenso sostenido de la mortalidad por homicidios, aunque no de manera homogénea. Esto es, si bien del 2002 al 2011 se presentó una disminución de la mortalidad por homicidios en todos los estratos, esta disminución fue más acentuada en el Medio y en el Bajo (con un cambio relativo de 69,4 y $59 \%$, respectivamente); mientras que la menor disminución se presentó en el estrato Muy Alto (solamente un 23,3\%).

Por sexo, se observa una situación análoga, ya que los estratos que tuvieron los mayores descensos de la tasa de homicidios fueron el
Medio (69,5\% en hombres y 65,8\% en mujeres) y el Bajo (65,8 y 56,9\% para hombres y mujeres, respectivamente). De igual forma, el estrato Muy Alto tuvo la menor disminución de todos los estratos considerados (solo $24,9 \%$ en hombres) y resalta que en mujeres es el único que presentó un incremento de la tasa en ese periodo (de 4,6 a 4,8 homicidios). Una característica adicional de la tendencia de homicidios por estrato, en ambos sexos, es que las tasas de mortalidad tienden a converger hacia un mismo nivel a medida que pasa el tiempo (figuras 2 y 3 ).

En cuanto a los AVP por estrato, en el 2000 el estrato de Bajo INB tenía el mayor nivel de AVp $(0,58)$, mientras que el de Muy Alto INB presentó el menor $(0,44)$; esto tanto para hombres (figura 4) como para mujeres (figura 5). La situación se modifica en el 2002, ya que el estrato Medio se ubicó como aquel con mayores AVP, presentando un cambio porcentual de $24,8 \%$ con respecto al 2000 (con un aumento de $17,4 \%$ para hombres y $76,5 \%$ en mujeres), cifra que es la mayor entre los estratos analizados. Cabe resaltar que el estrato Muy Alto presentó un incremento significativo de $32 \%$ en el caso de mujeres. Del 2002 al 2011

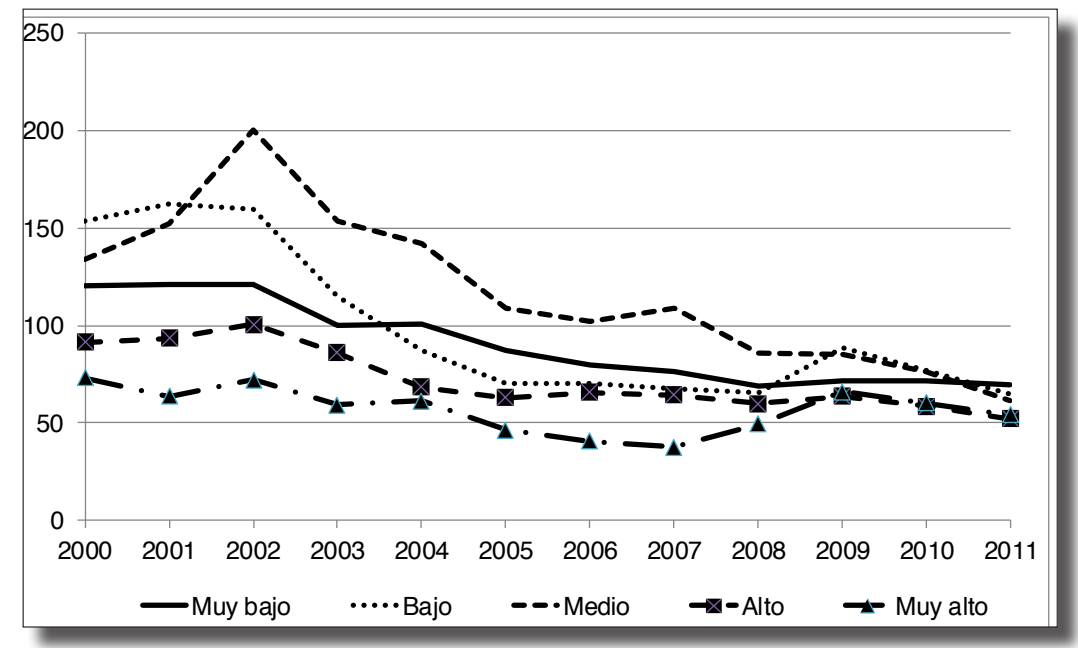

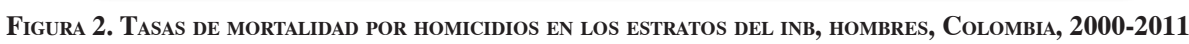
Fuente: elaboración propia con base en datos del DANE, 2000-2011 


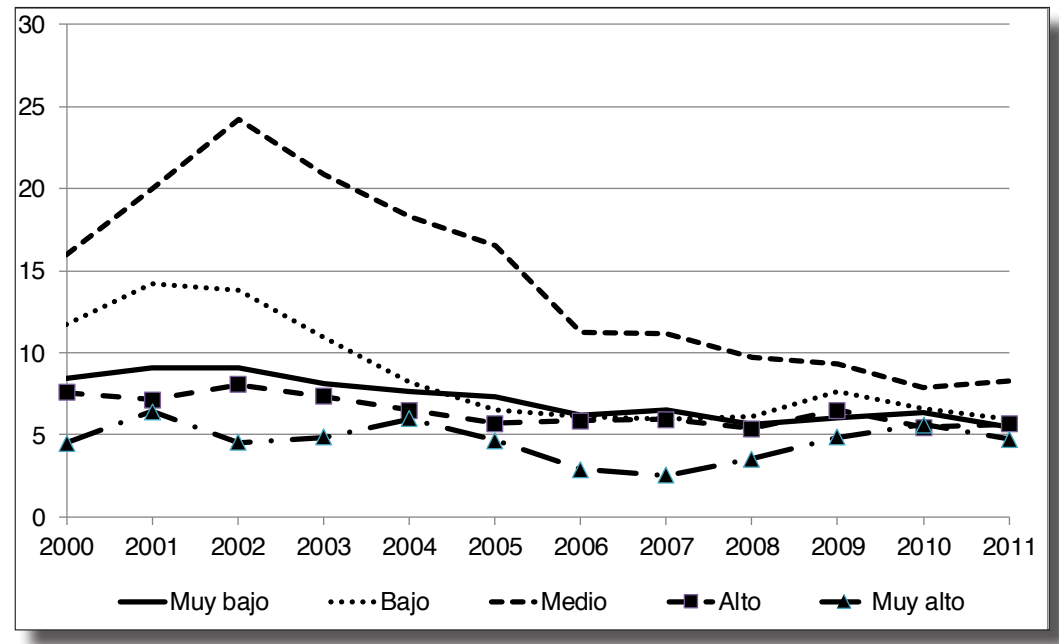

Figura 3. Tasas de mortalidad por homicidios en los estratos del inb, mujeres, Colombia, 2000-2011 Fuente: elaboración propia con base en datos del DANE, 2000-2011

se observa una reducción del impacto de la mortalidad por homicidios en la población de todos los estratos, con el descenso más importante en el estrato Medio $(42,4 \%)$; situación similar ocurre en hombres y mujeres, para los cuales este estrato tuvo el descenso más significativo en ese periodo ( $38,7 \%$ y $51 \%$, respectivamente), aunque destaca el estrato Bajo en mujeres con un descenso importante de $36,2 \%$. Resalta la tendencia del estrato Muy Alto, el cual tuvo una disminución importante del nivel de homicidios desde el 2002 hasta el 2007, pero a partir de ese año la tendencia se invierte, presentándose un incremento significativo del 44,8\% (33,8\% para hombres y un $135,4 \%$ para mujeres). Debido a ello se recomienda analizar las razones del repunte de este estrato, especialmente para mujeres, ya que fue el único estrato que en todo el periodo

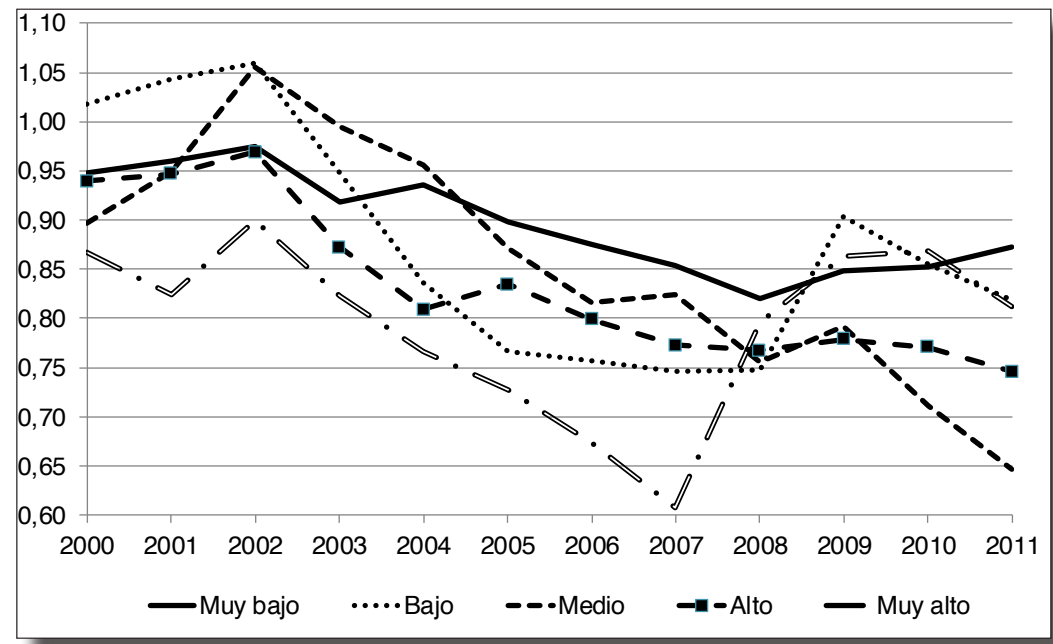




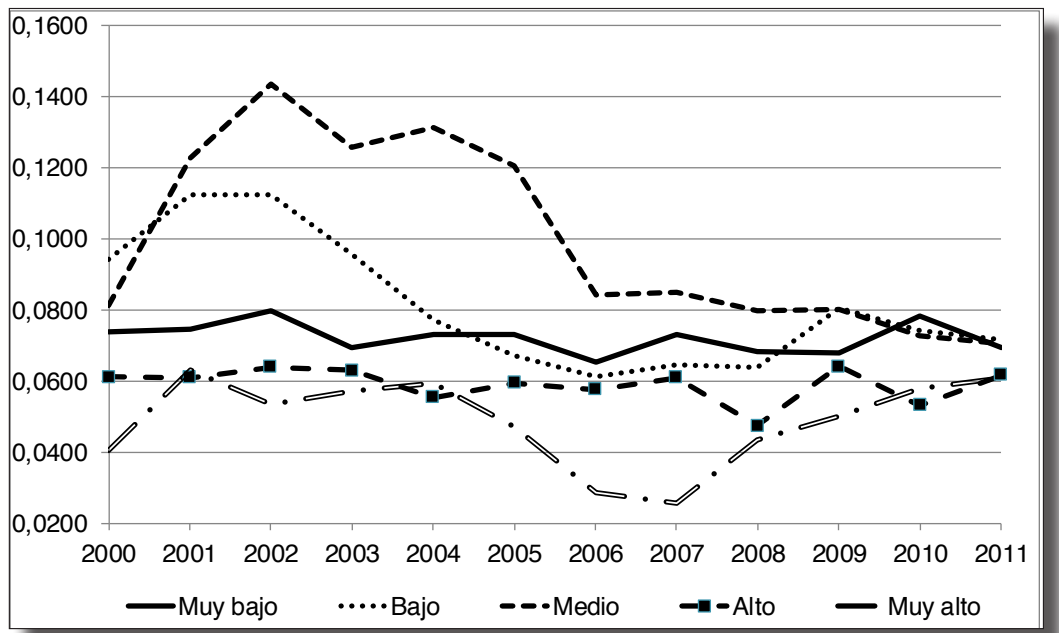

Figura 5. Años de Vida Perdidos por homicidios en los estratos del inb, Mujeres Colombia 2000-2011

Fuente: elaboración propia con base en datos del DANE, 2000-2011

analizado presentó un incremento de la mortalidad $(50,7 \%)$.

A diferencia de las tasas de mortalidad por estrato, que hasta cierto punto tienden a converger en ambos sexos, cuando se analizan los AVP se tiene una situación diferente. Si bien para mujeres se da dicha convergencia del nivel de la mortalidad analizada por medio de los AVP, en el caso de hombres no se presenta este fenómeno (figuras 4 y 5). Esta situación puede deberse a que la mortalidad femenina por homicidios, en la mayoría de las edades, incluidas las consideradas por los AVP, presenta una tendencia a asemejarse entre los distintos estratos. Sin embargo, en el caso de hombres, la diferencia de los resultados entre las tasas y los AVP indican que en el rango de edad de 15 a 49 años no se presenta esta convergencia del nivel de la mortalidad, a pesar de que en las edades no incluidas en este rango sí existe dicha tendencia.

\section{Discusión}

La violencia es reconocida como uno de los principales problemas de salud pública
(26), debido a que tiene una alta carga de mortalidad y morbilidad, requiere cuidados médicos, psicológicos o de rehabilitación y perjudica tanto a la víctima como a su familia y a la sociedad entera, afectando negativamente el desarrollo social y económico de los países $(27,28)$. Los estudios alrededor del tema de la violencia homicida en Colombia han mostrado la importancia de la discusión con sustento empírico y la utilización de herramientas metodológicas sólidas (16) para conocer el impacto de la mortalidad por homicidios en diferentes grupos de edad, por sexo, geográficamente o por distintas características socioeconómicas, lo que puede proveer valiosa información para determinar la forma más eficiente de combatir la violencia (29).

Los resultados de este estudio permiten corroborar el descenso de la mortalidad por homicidios que se presentó en Colombia durante la última década, el cual se presenta después de un periodo en el cual el país alcanzó las tasas de homicidios más altas del mundo (8). La tendencia analizada de ambos indicadores utilizados mostró un incremento significativo de la mortalidad por esta causa entre el $2000 \mathrm{y}$ el 
2002, también detectado por Moreno y Cendales (3) y Barón (29), pero en esta investigación se observó desde ese año una notable reducción de la mortalidad por homicidios, al igual que lo encontrado por Moreno y Cendales (3), Briseño (8), Salama (9), Barón (29) y CubidesSalazar (30). Sin embargo, este descenso ha sido incompleto ya que no se ha presentado de manera homogénea a nivel regional o por grupos de edad y continúa en niveles altos con respecto a otros países de la región y del mundo (31). Resalta el ligero repunte de las tasas en el 2009, tanto en hombres como en mujeres, cuyo análisis escapa los objetivos de este texto. Estos resultados son similares a los encontrados en otras investigaciones sobre el tema $(3,8,9,29,32)$.

Es importante mencionar que si bien se ha presentado un descenso de la mortalidad por homicidios en los últimos años, el cambio relativo de las tasas de mortalidad fue significativamente mayor, mientras que los AVP en el grupo de edad de 15 a 49 años se redujeron en un monto menor en términos relativos. Como se mencionó previamente, esta situación se puede deber principalmente a que el descenso de la mortalidad se presentó en edades mayores a 35 años y no tanto en las edades más jóvenes. Estos resultados proveen de información necesaria para identificar oportunidades sobre las cuales se base el diseño y la implementación de políticas públicas dirigidas a reducir el impacto de la mortalidad por esta causa.

Los resultados de los AVP permiten además confirmar que existen dos características que muestran marcadas diferencias en la incidencia de homicidios: el sexo y la edad (8). El sesgo de sexo es muy importante, pues tanto las víctimas como los victimarios son mayoritariamente hombres $(8,33)$. En el 2011, el 91\% de las víctimas correspondió a hombres, lo que ubica a la población masculina como principal protagonista de este fenómeno, con efectos directos sobre la estructura demográfica y, por consiguiente, una disminución de la proporción de población económicamente activa, lo cual incide probablemente en una mayor pobreza y menos oportunidades para la población dependiente, en particular niños y adultos mayores (18). La segunda característica se relaciona con la edad y se ha estudiado que tanto las víctimas como los perpetradores son en su mayoría jóvenes entre 15 y 30 años de edad (8), por lo que este grupo de edad se puede considerar como el más vulnerable a la violencia homicida $(2,17,28,34,35)$.

La tendencia observada de la mortalidad por homicidios en Colombia en la última década lleva inevitablemente a indagar sobre cuáles han sido los principales factores asociados con su incidencia. Algunas investigaciones han encontrado en el aspecto económico una explicación a la violencia homicida en Colombia; específicamente, argumentan que es en la distribución arbitraria y desigual de la rique$\mathrm{za}$, donde la violencia encuentra el espacio propicio para su desarrollo, constituyéndose por tanto en determinantes importantes de la violencia homicida en Colombia $(4,15)$. Si bien la evidencia empírica sobre la relación entre factores objetivos - como la pobreza, la desigualdad y la exclusión - y la violencia homicida no es sencilla de interpretar, algunos estudios comparativos han mostrado que existe cierta relación entre estos factores $(4,16)$, e incluso otros han concluido que el aspecto económico tiene un efecto causal sustancial sobre la cantidad de homicidios que se cometen en Colombia (36).

Ahora bien, en Colombia la pobreza ha sido medida desde finales de los años ochenta con la metodología de Necesidades Básicas Insatisfechas (19). En este caso, la pobreza es entendida como privación de capacidades básicas $(37,38)$ y comprende las situaciones en las cuales se carece de las potencialidades 
específicas para la satisfacción de las necesidades básicas (19). En este estudio se buscó analizar la relación o asociación entre este índice y la mortalidad por homicidios, estratificando los departamentos en cinco grupos. En el análisis del impacto de la mortalidad por homicidios en los distintos estratos, destaca que el estrato Medio presentó los niveles más altos en el periodo considerado. Es importante resaltar también que el estrato Bajo presentó la disminución de mortalidad más importante, lo que probablemente es un reflejo de la disminución de la mortalidad por esta causa en Medellín, ciudad que alcanzó las mayores tasas en todo el país, pero que presentó un descenso abrupto de 1998 en adelante (12). Aunque el estrato Medio presentó el mayor descenso relativo de AVP entre los 15 y los 49 años, lo que implica que si bien en el primero se redujo la mortalidad por homicidios en todo el rango de edad, en el segundo el cambio se presentó principalmente en las edades jóvenes. Es importante mencionar que si bien Colombia ha tenido una violencia rural importante, esta tiende a ser un fenómeno particularmente urbano, ya que es en las ciudades donde se cometen la mayoría de los asesinatos (8). Resalta que en el 2011, el estrato con los mayores Avp fue el de Muy Bajo InB, lo que puede estar relacionado con la violencia urbana, especialmente en el Valle del Cauca, la cual continúa siendo una zona con altos índices de violencia (38). Por sexo, se tiene un panorama distinto, ya que el estrato Medio para mujeres continuó siendo el estrato con mayor nivel de AVP durante todo el periodo analizado, mientras que en el caso de hombres en el 2011 alcanzó el menor nivel de AVP entre todos los estratos. Asimismo, los estratos de Alto y Muy Alto INB presentaron la menor mortalidad en mujeres. En el caso de los hombres la situación fue análoga hasta el 2008, pero a partir de ese año el estrato Muy Alto presentó un incremento significativo de la mortalidad por esta causa. Otra característica del estrato más pobre es que presentó la menor disminución relativa de la mortalidad por esta causa. En el caso de las mujeres hasta presentó un incremento de poco más de $50 \%$ en los AVP y tuvo un repunte importante a partir del 2009 en el caso de los hombres, lo que lo llevó a situarse como el segundo estrato con mayor mortalidad al final del periodo de estudio, situación que se considera importante analizar en investigaciones futuras centradas en los departamentos que conforman dicho estrato.

Los resultados obtenidos en este estudio no solo permiten corroborar la tendencia decreciente de la mortalidad por homicidios en todo el país, analizada también en otros estudios sobre el tema $(3,16,29,39)$, sino que dan cuenta de la magnitud de su impacto diferencial por estrato, sexo y edad. Asimismo, resalta que parece existir una escasa relación entre el INB y la mortalidad por homicidios, lo que puede implicar una débil asociación entre la pobreza, medida a partir del INB, y la violencia. Esto, por tanto, lleva a pensar que de existir una relación causal entre la pobreza y la violencia, todavía no se conocen bien los mecanismos a través de los cuales estas se asocian entre sí. Es posible que parte de la respuesta se encuentre en que la pobreza influye en la criminalidad y en los homicidios, pero no de manera directa, sino mediadas por el fortalecimiento o debilitamiento del orden normativo formal e informal que sirve de contención a los comportamientos violentos (8). Es por ello que las políticas públicas encaminadas a reducir la violencia y la criminalidad, su aplicación y éxito estarán mediados tanto por el nivel de impunidad en la sociedad como por el refuerzo general del sentido de la norma y del incremento de la cohesión social (8).

Se podría argumentar, por tanto, que la pobreza no es condición necesaria de la violencia homicida, porque esta puede ser producida por otras razones, y tampoco 
se puede considerar condición suficiente, ya que no conduce inevitablemente a ella porque no todos los grupos sociales pobres presentan altas tasas de homicidios (16), lo que no implica que no exista cierta relación entre estos fenómenos.

Una explicación alternativa de la violencia en el caso colombiano se basa en que la presencia y la actividad de grupos armados irregulares - como narcotraficantes, guerrillas y paramilitares-, así como la debilidad y un deficiente desempeño del sistema de justicia, tienen una relación positiva con la violencia homicida $(16,40)$. Se argumenta entonces que estos factores influyen en el incremento de los crímenes violentos, ya que sus dinámicas están en la raíz de los enfrentamientos relacionados con el control de mercados y sectores (34). Por tanto, una explicación del descenso observado de las tasas de mortalidad en el periodo 2002-2008 puede estar relacionada con el impacto del desmantelamiento de los carteles de la droga en Cali y Medellín, así como el papel de iniciativas de reducción de la violencia, tanto de orden nacional como local, y la desmovilización de grupos de paramilitares (31). Sin embargo, después de este descenso, se pudo observar un repunte de la tasa de homicidios y de los AVP entre el 2009 y el 2010, acercándose al nivel del promedio histórico, el cual ha sido atribuido a un incremento de la violencia en las ciudades y regiones donde el negocio del narcotráfico generaba la mayor parte de los ingresos del crimen organizado, y donde contaba con más recursos (41). Es importante recalcar que a pesar de la importancia del narcotráfico, la guerrilla, los paramilitares y el colapso de la justicia como elementos vitales para explicar la violencia explosiva de finales de los noventa, y la posterior caída del nivel de los homicidios en los últimos años, estos no ocurren en un vacío social, económico y político, sino que son eslabones de una cadena causal más larga, de la que la pobreza, la desigualdad y la exclusión también son parte (16).

Otro aspecto que se argumenta que está relacionado con la violencia homicida, es la exclusión política, social y económica (15). Esto es, a través de la distribución inequitativa de la riqueza, del ingreso y de las oportunidades se produce la exclusión de amplios grupos y regiones de la población en las decisiones políticas, económicas y sociales (40), lo cual, en un contexto de debilidad del Estado, incapaz de asumir sus funciones básicas de legalidad, de mediación en los conflictos, de provisión del bienestar colectivo y de formación de ciudadanía, tiene elevadas posibilidades de motivar el conflicto y la violencia (15). Si bien es una cuestión compleja, y todavía incompleta, entablar una relación causal directa entre la exclusión y la violencia, es importante considerarla como un factor que podría favorecer la aparición de conflictos violentos a partir de la necesidad de supervivencia de grupos marginados de la población que se sienten oprimidos por el orden social excluyente (12).

Una explicación adicional es aquella que identifica junto con la pobreza, la desigualdad, la exclusión política, social y económica, a una arraigada y extendida cultura de intolerancia y violencia como variable explicativa crucial en la incidencia de homicidios en Colombia (16). Se puede argumentar, por lo tanto, que ni la pobreza, ni las desigualdades, ni otras razones estructurales o transversales pueden por sí solas responder por el nivel de la violencia colombiana (42). Esto se cree que está relacionado con el hecho de que en las ciencias sociales es difícil encontrar un fenómeno que exhiba una causalidad simple, en términos de la necesidad o la suficiencia de un puñado de variables para dar un resultado determinado (16). 
Se puede concluir, por tanto, que la violencia en general, y más una violencia homicida con las características como la colombiana, es el resultado de un complejo número de causas. Es por ello que en la búsqueda de las principales causas de la violencia homicida en Colombia se deben señalar aspectos como la pobreza, la desigualdad, la exclusión social, educativa y política, conjuntamente con la debilidad del Estado y del sistema judicial, los altos grados de impunidad frente al crimen, el resquebrajamiento de valores como el respeto al derecho de los demás, la solidaridad, la corrupción, la rápida urbanización sin planificación y los acelerados cambios demográficos $(4,8,36)$, un fenómeno cultural generalizado enraizado en las características de la sociedad $(16,43,44)$, así como aspectos de índole política como la implementación del Plan Colombia, el incremento de la movilidad y la efectividad de las fuerzas armadas, así como el desmantelamiento de los cárteles y la reducción de la presencia de cultivos de cocaína (que se traduce en menos recursos financieros que van a grupos criminales, menos intimidación y menos actividades que pueden escalar a homicidio) (29).

Debido a esta multicausalidad del fenómeno de la violencia, su estudio no debe ser llevado a cabo desde una sola disciplina o perspectiva, sino que es indispensable contar con investigaciones que permitan obtener una visión integral del fenómeno que posibilite el estudio y la comprensión de sus intrincadas dinámicas (40). Este reconocimiento del carácter multidimensional de la violencia es una forma de reconocer su complejidad, por tanto, se recomienda que su análisis se aborde desde diversas disciplinas.

Un último aspecto que tomar en cuenta y que ha sido poco investigado, es que la violencia constituye un problema muy serio de salud pública, no solo por la elevada mortalidad que ocasiona, sino también por la discapa- cidad y el elevado costo resultantes de las lesiones no mortales (45). Esto es, las muertes por violencia constituyen solamente una pequeña proporción del problema, ya que de acuerdo con estimaciones de la Organización Mundial de la Salud, por cada persona que muere de una lesión traumática, hay 15 que quedan gravemente afectados y otros 30 a 40 que registran daños que requieren cuidados médicos, psicológicos o de rehabilitación (18). Esto conlleva elevados costos sociales y económicos, con implicaciones negativas para los sistemas de salud, las víctimas, sus familias, el sector productivo y la sociedad en general (46).

\section{Limitaciones}

En esta investigación la información sobre la violencia se centró en los homicidios, por dos motivos principales: la gravedad del acto y que son el tipo de violencia para el cual se dispone de estadísticas más fiables, porque suelen registrarse más acuciosamente $(3,4,20)$. Sin embargo, es importante señalar que en Colombia el sistema de información de las estadísticas sobre mortalidad y morbilidad presenta un subregistro significativo de las muertes ocurridas, especialmente por hechos violentos (39), ya que las muertes ocurridas durante enfrentamientos armados, o los homicidios a manos de grupos ilegales, podrían no estar contabilizadas por diversas circunstancias, quedando fuera del alcance de los registros nacionales de mortalidad (3). Por consiguiente, las defunciones por homicidios analizadas aquí, es posible que tengan cierto grado de subregistro, lo que representa una limitación importante para el estudio.

Las estadísticas vitales de mortalidad, en general, en los países de la región tienen además otro tipo de limitaciones, como la falta de uniformidad en las definiciones del registro, una cobertura inadecuada en regiones poco accesibles, errores de omisión, subregistro y 
largos procesos burocráticos que retrasan la publicación de la información (47).

Otra limitante del estudio se relaciona con el uso del indicador de INB, ya que la forma categórica en que se calcula tiende a no incluir personas que, si bien pueden no tener satisfecha una de las cinco características elegidas, pueden tener altos niveles de insatisfacción en las restantes, lo que se refiere a que el método no provee una variable continua donde los distintos indicadores tengan una ponderación particular, la cual es considerada como la principal limitación del método de Necesidades Básicas Insatisfechas (19).

Una limitante adicional, es que la estimación de los AVP se ve restringida al tener que delimitarse un rango de edad, en este caso en particular entre los 15 y 49 años, lo que provoca que se subestime el impacto total de la mortalidad por homicidios, el cual puede ser más alto que el establecido en este trabajo. Sin embargo, la mayor parte de individuos cuya muerte se debió a esta causa quedó incluida en esta investigación.

\section{Recomendaciones}

El comportamiento de la mortalidad por homicidios en Colombia durante los primeros años del siglo XXI es un fenómeno de gran relevancia, pero para el cual aún no se tiene una explicación satisfactoria, por lo que se tienen que realizar más investigaciones sobre el tema, tomando en cuenta su carácter multicausal y multifactorial. Esto es, conocer más acerca del impacto de la mortalidad por homicidios es un paso imprescindible para "pasar de la preocupación a la acción en esta materia" (34), y a su vez, proveer los insumos necesarios para la elaboración de políticas de prevención a la medida de las necesidades de cada departamento del país. Esto debido a que el análisis de la mortalidad en general, y de la mortalidad por homicidios en particu- lar, permite profundizar en el conocimiento de la situación social, económica, política y de salud en una sociedad (48).

\section{Referencias bibliográficas}

1. López M, Híjar M, Rascón R, Blanco, J. La violencia y sus repercusiones en la salud; reflexiones teóricas y magnitud del problema en México. Salud Pública Mex. 1997; 39 (006): 565-72.

2. Organización Panamericana de la Salud. Informe Mundial sobre la violencia y la salud: Resumen. Washington D.C.: ops/OMs; 2002.

3. Moreno C, Cendales R. Mortalidad y años potenciales de vida perdidos por homicidios en Colombia, 1985-2006. Rev Panam Salud Pública. 2011; 30 (4): 342-53.

4. Cardona M, García HI, Giraldo CA, López MV, Suárez CM, Corcho DC et al. Homicides in Medellín, Colombia, from 1990 to 2002: Victims, Motives and Circumstances. Cad. Saúde Pública. 2005; 21 (3): 840-51.

5. Short JJ. Poverty, Ethnicity and Violent Crime. Boulder: Westview Press; 1997.

6. Souza, E. Homicídios: Metáfora de uma Nação Autofágica [Tese de Doutorado]. Rio de Janeiro: Escola Nacional de Saúde Pública, Fundação Oswaldo Cruz; 1995.

7. United Nations Office on Drugs and Crime. 2013 Global Study on Homicide. Vienna: UNODC; 2013.

8. Briceño-León R. La comprensión de los homicidios en América Latina: ¿Pobreza o Institucionalidad? Ciênc. Saúde Colet. 2012; 17 (12): 3159-70.

9. Salama P. Homicidios, ies ineluctable la violencia en América Latina? Front. Norte. 2013; 25 (49):7-27.

10. Rodríguez J, Gallardo HM. Mortalidad y años de vida ajustados por discapacidad como medidas de la carga de enfermedad: Colombia, 1985-1995. Colombia: Ministerio de Salud; 1999.

11. Bonilla-Mejía L. Demografía, juventud y homicidios en Colombia, 1979-2006. Lecturas de Economía. 2010; 72: 103-140.

12. García HI, Giraldo CA, López MV, Pastor MDP, Cardona M, Tapias CE et al. Treinta años de homicidios en Medellín, Colombia, 1979-2008. Cad Saúde Pública. 2012; 28 (9): 1699-712.

13. Cotte A. Crecimiento, desigualdad y pobreza: un análisis de la violencia en Colombia. Revista de Investigación. 2006; 6 (002): 209-222.

14. Parra C. Determinantes de la inversión en Colombia: Evidencia sobre el capital humano y la violencia. Archivos de Macroeconomía. 1998; 84: 1-34.

15. López C, García A. Los costos ocultos de la paz en Colombia. En: Solimano A, Sáez F, Moser C, López C, editores. Ensayos sobre paz y desarrollo. El caso de Colombia y la experiencia internacional. Colombia: The World Bank; 1999. pp. 81-178. 
16. Valenzuela P. Reflexiones sobre interpretaciones recientes de la violencia en Colombia. Revista Reflexión Política. 2002; 4 (8): 125-46.

17. Rubio M. Violencia y conflicto en los noventa. Revista Coyuntura Social. 2001; 22 (1): 11-32.

18. Cardona D, Segura ÁM, Espinosa A, Segura A. Homicidios y suicidios en jóvenes de 15 a 24 años, Colombia, 1998-2008. Biomédica. 2013; 33 (4): 574-86.

19. Fresneda O. La medida de necesidades básicas insatisfechas (NBI) como instrumento de medición de la pobreza y focalización de programas. Serie Estudios y Perspectivas (Cepal). 2007; 18: 1-193.

20. Comisión Económica para América Latina y el Caribe (Cepal). Agenda social. Seguridad ciudadana y violencia. Panorama Social de América Latina 1998; 1999.

21. Arriaga E. Comentarios sobre algunos índices para medir el nivel y el cambio de la mortalidad. Estudios Demográficos y Urbanos. 1996; 11 (1): 5-30.

22. Arriaga E. Los años de vida perdidos: Su utilización para medir el nivel y cambio de la mortalidad. Notas de Población. 1996; 24 (63): 7-38.

23. González-Pérez G, Vega-López M, Cabrera-Pivaral C. Impacto de la violencia homicida en la esperanza de vida masculina de México. Rev Panam Salud Publica. 2012; 32 (5): 335-42.

24. García P. Indicadores sociales departamentales. En: Departamento Nacional de Planeación, editor. Colombia: Boletines Sistema de Indicadores Sociodemográficos; 2007.

25. Dávila-Cervantes CA, Pardo-Montaño AM. Magnitud y tendencia de la mortalidad por homicidios en Colombia y México, 2000-2011. Rev Panam Salud Publica. 2014; 36 (1): 10-6.

26. Krug EG, Dahlberg LL, Mercy JA, Zwi AB. Informe mundial sobre la violencia y la salud. Rev Inst Med Trop S Paulo. 2003; 45 (3): 130.

27. Concha A. Impacto social y económico de la violencia en las Américas. Biomédica. 2002; 22 (su2): 347-61.

28. Rodríguez M. Evolución de la mortalidad por homicidio en Medellín (Colombia), 1975- 2003. Gac Sanit. 2005; 19 (3): 238-41.

29. Baron JD. El homicidio en los tiempos del Plan Colombia. Cartagena: Banco de la República; 2009.

30. Cubides-Salazar OM. La violencia del narcotráfico en los países de mayor producción de coca: los casos de Perú y Colombia. Papel Político. 2014; 19 (2): 657-90.

31. Aguirre K, Restrepo JA. El control de armas como estrategia de reducción de la violencia en Colombia: pertinencia, estado y desafíos. Rev Crim. 2010; 52 (1): 265-84.

32. Echeverry JC. Lessons from Colombian Economic Development. Bogotá: Universidad de los Andes, Facultad de Medicina, Centro de Estudios sobre Desarrollo Económico; 2009.
33. Rosselli D, Tarazona N, Aroca A. La salud en Colombia 1953-2013: Un análisis de estadísticas vitales. Medicina. 2014; 36 (2): 120-35.

34. Banco Interamericano de Desarrollo. Análisis de los homicidios en seis países de América Latina. Washington D.C.: Inter-American Development Bank; 2013.

35. Stevenson G. Gender Inequality, Concentrated Disadvantage and Homicide Victimization: A Sex and Race Specific Analysis of Homicide Victimization Rates in large U.S Cities [tesis de doctorado]. Louisiana: Lousiana State University; 2003.

36. Carranza JE, Dueñas J, González CG. Análisis empírico de la relación entre la actividad económica y la violencia homicida en Colombia. Estudios Gerenciales. 2011; 27 (119): 59-77.

37. Sen A. Desarrollo y libertad. Bogotá: Planeta; 2000.

38. Álvarez C, González C. Análisis espacial de la violencia homicida en el pacífico colombiano. Econ Adm. 2012; 9 (1): 69-85.

39. Otero A. Diferencias departamentales en las causas de mortalidad en Colombia. Documentos de Trabajo sobre Economía Regional del Banco de la República de Colombia. 2013; 187: 1-44.

40. Martínez A. Análisis económico de la violencia en Colombia. Una nota sobre la literatura. Cuadernos de Economía Universidad Nacional. 2001; 20: 1-34.

41. Salazar B, Frasser C. Menos cocaína, más violencia. Revista de Economía Institucional. 2013; 15 (29): 327-34.

42. Gaitán F, Deas M. Dos ensayos especulativos sobre la violencia en Colombia. Bogotá: Fonade; 1995.

43. Becerra M, Sarmiento A. Análisis de las relaciones entre violencia y equidad. Bogotá: Departamento Nacional de Planeación; 1998.

44. Fajnzylber P, Lederman D, Loayza N. Crimen y violencia en América Latina. Bogotá: Editorial Alfaomega; 2001.

45. Cardona D, Escané G, Fantín MA, Peláez E. Mortalidad por causas externas: un problema de salud pública. Argentina, Chile y Colombia. 20002008. Población y Salud en Mesoamérica. 2013; 10 (2): 1-13.

46. Macías G, Almeida F, Naomar, Alazraqui M. Análisis de las muertes por accidentes de tránsito en el municipio de Lanús, Argentina, 1998-2004. Salud Colectiva. 2010; 6 (3): 313-328.

47. Hernández-Bringas H, Narro-Robles J. El homicidio en México, 2000-2008. Papeles de Población. 2010; 16 (63): 243-71.

48. Cárdenas R. Mortalidad: niveles, cambios y necesidades en materia de política pública. En: Rabell C, editora. Los mexicanos: un balance del cambio demográfico. México: Fondo de Cultura Económica; 2014. pp. 121-42. 